\title{
Symbol Synchronization in Coded UWB Systems using Adaptive Iterative Detection
}

\author{
Durai Thirupathi and Keith M. Chugg \\ Communication Sciences Institute \\ Dept. of Electrical Engineering-Systems \\ University of Southern California, Los Angeles 90089-2565 \\ \{thirupat, chugg\}@usc.edu
}

\begin{abstract}
Ultra-wide band (UWB) communication systems are extremely power limited systems typically with wide bandwidth to spare. The most common UWB system found in the literature, the impulse radio, uses very narrow pulses as a way to expand the bandwidth of the information bearing signal. For this reason, such systems are very sensitive to instabilities in the clock circuits. Therefore, extremely stable clocks are required for proper operation. In this paper, we propose novel techniques to trade off the clock stability with baseband digital processing in a coded UWB system. By jointly tracking the jitter and decoding the code, using adaptive iterative detection, we show that the specification on the clocks can be relaxed by orders of magnitude without significantly degrading the performance.
\end{abstract}

\section{INTRODUCTION}

After the discovery of turbo codes [1], iterative detection (ID) has become such a powerful tool that it is being used in various applications [2]. The basic principle behind ID is to exchange the so-called soft information (extrinsic information) among different modules that are essentially the probabilistic inverses of their counter-parts at the transmission/propagation end. Adaptive iterative detection (AID) [3], an extension to ID, was developed for cases where the detector has to deal with the presence of unknown, possibly time-varying parameters. The unknown parameters could either be deterministic or probabilistic. This paper examines the application of AID to symbol synchronization in coded ultra-wide band (UWB) systems.

In an UWB communication system that uses sub-nano second pulses to communicate, clock stability is one of the crucial elements to the system design. Highly stable clocks are needed to control both the sampling and the integrate and dump circuits. Typically, the quality of an oscillator is specified by the phase noise (in the spectral domain) or the cycle-to-cycle jitter (in the time domain). The higher the phase noise, the lower the stability of the oscillator and hence lower the typical cost. In this work, we attempt to relax the specifications on the oscillators that can be used in the UWB system under the assumption that channel coding is used (i.e., the system under consideration is a coded UWB system). In order to achieve this goal, we exploit the structure of the channel code and apply the principle of iterative detection between the decoder and the tracking device. The phase noise process in the oscillator is modelled as first order Gauss-Markov [4].

Quantization based estimation (QBE) is an estimation technique that has recently been proposed for estimation of unknown parameters that are typically non-linear in the obser-

This work was supported in part by the ARO under the grant DAAD19-01-10477 . vation [6]. The basic idea behind this approach is to approximate the model (typically continuous) that corresponds to the unknown variable such that it can take only finite number of values (discrete) at any given time. We propose to use this estimation technique to estimate jitter, since, as shown later, the timing jitter is non-linear in the observation. In our case, the jitter model, namely, the discrete time, continuous Markov process is approximated by a Markov chain.

The rest of the paper is organized as follows. Section II describes the system model. QBE as applied to tracking jitter is explained in Section III, along with two different algorithms that can be used to estimate the jitter. Numerical results and conclusions are given in Sections IV and V, respectively.

\section{System Model}

The transmitter section of interest is shown in Figure 1a. It consists of an independent, identically distributed (i.i.d.) source that is encoded by a channel code, in our case a turbo-like code. The output of this encoder is interleaved before being fed to a pulse shaping circuit. The modulation is assumed to be antipodal, bi-phase (flip) modulation. The frame period $\left(T_{f}\right)$ is assumed to be much larger than the pulse duration $\left(T_{p}\right)$. The number of pulses per uncoded data bit $(\eta)$ is also assumed to be a large integer.

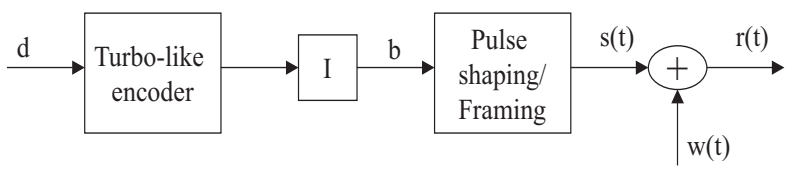

(a)

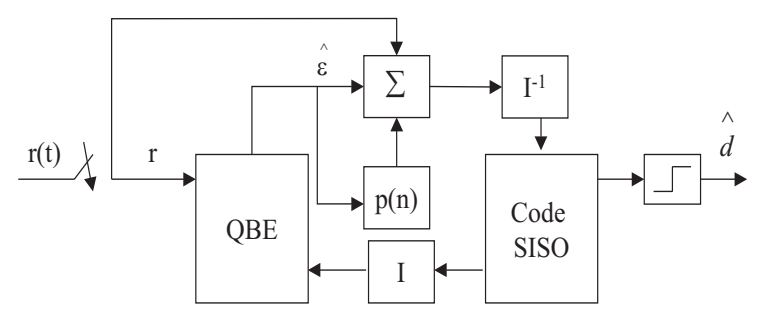

(b)

Fig. 1. A section of the UWB transceiver under consideration. (a) Transmitter (b) Receiver. 
The transmitted signal can be written as,

$$
s(t)=\sum_{j} A_{j} b_{j} p\left(t-j T_{f}-\tau_{j}\right)
$$

where $b_{j} \in\{+1,-1\}$ represents the $j$-th coded symbol and $A_{j}$, the corresponding amplitude. The parameter $\tau_{j}$ characterizes the timing uncertainty in the pulse shaping circuit corresponding to the $j$-th frame. The signal is transmitted through an additive white Gaussian noise (AWGN) channel. The received band-limited signal can be written as,

$$
r(t)=\sum_{j} A_{j} b_{j} p\left(t-j T_{f}-\epsilon_{j}\right)+w(t),
$$

where $\epsilon_{j}$ characterizes the total timing jitter (corresponding to the $j$-th frame) in the link with reference to the receiver clock. Under the assumption that the sampling circuit is free of any jitter $^{1}$, the jitter can be modelled as first order Gauss-Markov such that

$$
\epsilon_{j}=\alpha \epsilon_{j-1}+u_{j},
$$

where $u_{j} \sim N\left(0, \sigma_{u}^{2}\right)$ and uncorrelated in time. The scaling constant $\alpha$ is a modelling parameter. The sampled signal can be written as,

$$
r\left(n T_{\text {samp }}\right)=\sum_{j} A_{j} b_{j} p\left(n T_{s a m p}-j T_{f}-\epsilon_{j}\right)+w\left(n T_{s a m p}\right),
$$

where $T_{\text {samp }}$ corresponds to the inverse of the sampling frequency. The over-sampling factor $N$, with respect to the pulse duration $T_{p}$, is assumed to be much greater than 2, i.e., there are more than 2 samples per pulse. The samples that correspond to the $i$-th pulse can be compactly represented by a vector as,

$$
\underline{r}_{i}=[r(N i) r(N i+1) \ldots r(N(i+1)-1)]^{t},
$$

where the notation $[\cdot]^{t}$ is used to denote the transpose of a vector. Given this sampled sequence, the goal is to derive a 'good' data detection scheme. There are two approaches for this problem. One is to derive an estimator-correlator (EC) type receiver [2, Ch. 4] where estimation of the unknown parameters and data detection are performed jointly. Since the unknown jitter is random and non-linear in the observation model, typically, the estimator would be an extended-Kalman filter. Though it is not optimal, this approach yields very good performance. However, the complexity of such a joint approach is prohibitive. Hence, in this paper, the problem is decoupled into estimation of the jitter and using the estimates to obtain soft information on both the coded symbols and the data. After iterating between the estimator and the data detector (decoder), hard decisions are made on the data. Since the jitter is assumed to be within a small range, the estimation of this parameter is referred to as tracking in this work [5].

\footnotetext{
${ }^{1}$ In the presence of timing jitter in the sampling circuit, that uncertainty can also be lumped into the total timing jitter $\epsilon_{j}$.
}

\section{QuAntization BASED Estimation (QBE) AND DECODING}

QBE is an estimation technique that has been used in estimating unknown phase, frequency, etc. [6]. This technique was developed to estimate unknown parameters that are non-linear in the observation. The basic idea behind this technique is to approximate the model of the unknown parameter that takes on values on a continuum, by a discrete model. The term 'quantization' in QBE refers to this approximation of the continuous model for the parameter by a discrete one. In our case, as given in (4), the unknown parameter, namely, the jitter, is nonlinear in the observation. Therefore, we propose to use the QBE technique to estimate the jitter. As mentioned earlier, the jitter model, which is a discrete time, continuous Markov process is approximated by a Markov chain. It should be noted that there is no physical quantizer present. The entire decoder structure including the QBE is shown in Figure 1b.

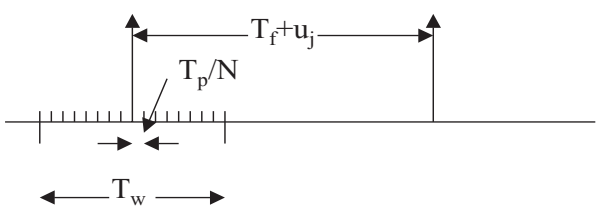

(a)

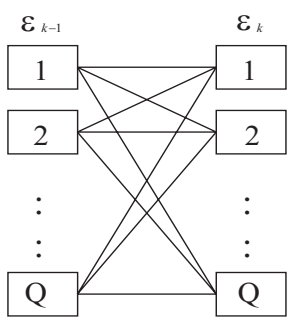

(b)

Fig. 2. (a) Relationship among different timing information in the QBE (b) Trellis structure of the quantization based estimator

The sampling circuit is activated for a small duration $T_{w}$ such that $T_{p}<T_{w}<<T_{f}$. The relationship among various timing information is shown in Figure 2a. Both the over-sampling factor $N$ and the sampling window duration $T_{w}$ are design parameters. The former depends on the precision with which the jitter needs to be estimated whereas the latter depends on the statistical characteristics of the timing jitter, typically the standard deviation of the cycle-to-cycle jitter. The larger the timing jitter to be tracked, the wider the sampling window should be. With the sampling interval being a fraction of $T_{p}, \frac{T_{p}}{N}$ to be precise, there will be $\frac{N T_{w}}{T_{p}}$ samples in the sampling window. Without loss of generality, we assume $\frac{T_{w}}{T_{p}}$ to be an integer. Since the random variable $\epsilon_{m}$ is assumed to be within $T_{w}$, sampling this space is equivalent to discretizing the values that $\epsilon_{m}$ can take. Thus, $Q=\frac{(N-1) T_{w}}{T_{p}}$ possible values can be modelled as pos- 
sible current states (at time $m$ ) for $\hat{\epsilon}_{m}$, the approximated value of $\epsilon_{m}$. The trellis structure of the estimator is shown in Figure 2b. Since the jitter is modelled as Gauss-Markov, the transition probabilities can be used to approximate the $a$-priori transition metric from the current state to the next state.

The basic function of the quantization based estimator (QBEr) is to produce estimates of the jitter based on the whole observation record $\underline{r}$. In other words, for time instant $m$, the output of the QBEr is

$$
\check{\epsilon}_{m}=\arg \max _{\hat{\epsilon}_{m}} \mathrm{P}\left[\hat{\epsilon}_{m}\right],
$$

where

$$
\mathrm{P}\left[\hat{\epsilon}_{m}\right]=\operatorname{Pr}\left(\hat{\epsilon}_{m} \mid \underline{r}\right),
$$

is the $a$-posteriori probability of the unknown jitter conditioned on the whole observation record. The conditional probability in (7) can be calculated in two different ways as described in the following. One way is to average out the jitter at all the locations other than location $m$, i.e., $\left\{\hat{\epsilon}_{i}\right\}_{i \neq m}$. In this case, the output of the QBEr can be written as,

$$
\begin{aligned}
\breve{\epsilon}_{m}= & \arg \max _{\hat{\epsilon}_{m}} \operatorname{Pr}\left(\hat{\epsilon}_{m} \mid \underline{r}\right), \\
= & \arg \max _{\hat{\epsilon}_{m}} \sum_{\hat{\epsilon}_{\hat{\epsilon}}: \hat{\epsilon}_{m}} \operatorname{Pr}(\underline{\hat{\epsilon}} \mid \underline{r}) \\
\equiv & \arg \max _{\hat{\epsilon}_{m}} \sum_{\underline{\hat{\epsilon}}: \hat{\epsilon}_{m}} \operatorname{Pr}(\underline{\underline{r}} \mid \underline{\hat{\epsilon}}) \operatorname{Pr}(\underline{\hat{\epsilon}}), \\
= & \arg \max _{\hat{\epsilon}_{m}} \sum_{\underline{\hat{\epsilon}}: \hat{\epsilon}_{m}}\left[\sum_{\underline{b}} \operatorname{Pr}(\underline{r} \mid \underline{\hat{\epsilon}}, \underline{b}) \operatorname{Pr}(\underline{b} \mid \underline{r}, \underline{\hat{\epsilon}})\right] \operatorname{Pr}(\underline{\hat{\epsilon}}), \\
\approx & \arg \max _{\hat{\epsilon}_{m}} \sum_{\underline{\hat{\epsilon}}: \hat{\epsilon}_{m}}\left[\sum_{\underline{b}} \prod_{i} \operatorname{Pr}\left(\underline{r}_{i}, \mid b_{i}, \hat{\epsilon}_{i}\right) \operatorname{Pr}\left(b_{i} \mid \underline{r}_{i}, \hat{\epsilon}_{i}\right)\right] \\
= & \arg \max _{\hat{\epsilon}_{m}} \sum_{\underline{\hat{\epsilon}}: \hat{\epsilon}_{m}}\left[\prod _ { i } \left(\sum_{b_{i}} \operatorname{Pr}\left(\underline{\hat{\epsilon}}_{i} \mid \hat{\epsilon}_{i-1}\right),\right.\right. \\
& \left.\operatorname{Pr}\left(\hat{\epsilon}_{i} \mid \hat{\epsilon}_{i-1}\right)\right] .
\end{aligned}
$$

The approximation in (8.5) is due to the fact that the joint distribution of the unknown parameters $\hat{\underline{\epsilon}}$ is approximated by the conditional distribution. Under the assumption that a large over-sampling factor $N$ is used, the transition probabilities $\operatorname{Pr}\left(\hat{\epsilon}_{i} \mid \hat{\epsilon}_{i-1}\right)$ can be calculated $a$-priori based on the model of $\epsilon_{i}$ as given in (3). The quantity $\operatorname{Pr}\left(b_{i} \mid \underline{x}_{i}, \hat{\epsilon}_{i}\right)$ is the $a$-priori information on the coded bits. This soft information can be obtained from the soft-in soft-out (SISO) module that corresponds to the channel code. The likelihood $\operatorname{Pr}\left(\underline{r}_{i} \mid b_{i}, \hat{\epsilon}_{i}\right)$ can be approximated as a Gaussian density. This is an approximation because instead of $\epsilon_{i}, \hat{\epsilon}_{i}$ is used in the likelihood calculation. The algorithm implied by (8) is referred to as the sum-product algorithm. In some cases, direct implementation of this algorithm might lead to numerical instability. Therefore, in practice, negative logarithm of (8) is implemented. This is referred to as the $\min ^{*}$-sum algorithm in the literature [2].
Another way of calculating the $a$-posteriori probability in (7) is to first find the sequence of jitter values $\underline{\hat{\epsilon}}$ consistent with $\hat{\epsilon}_{m}$ that maximizes the quantity $\operatorname{Pr}\{\underline{\hat{\epsilon}} \mid \underline{r}\}$. Expanding on this idea, the estimation rule implied by such approach can be written as,

$$
\begin{array}{r}
\breve{\epsilon}_{m}=\arg \max _{\hat{\epsilon}_{m}} \max _{\hat{\hat{\epsilon}}: \hat{\epsilon}_{m}}\left(\left[\sum _ { i } \left\{\sum_{b_{i}} \log \operatorname{Pr}\left\{\underline{r}_{i} \mid b_{i}, \hat{\epsilon}_{i}\right\}\right.\right.\right. \\
\left.\left.\left.\operatorname{Pr}\left\{b_{i} \mid \underline{r}_{i}, \hat{\epsilon}_{i}\right\}\right\}+\log \operatorname{Pr}\left\{\hat{\epsilon}_{i} \mid \hat{\epsilon}_{i-1}\right\}\right]\right) .
\end{array}
$$

The combining and marginalization algorithm implied by the above equation is known as the max-sum algorithm. When, instead of logarithm, negative logarithm is used, the algorithm is often referred to as the min-sum algorithm [2]. In the appendix, a connection is made between the maximum $a$-posteriori probability based expectation-maximization (MAP-EM) algorithm and the algorithm implied by (9).

\section{NumERICAL RESUlts}

Bit-error-rate (BER) is used as the performance measure of the proposed quantization based estimation scheme. The standard deviation of the independent noise component $u$ is assumed to be a fraction of the pulse duration $T_{p}$, where typically $T_{p} \leq 1 \mathrm{~ns}$. The root mean square (rms) variation in the output period of an oscillator due to this noise is referred to as cycle-tocycle jitter. Since the noise is independent from cycle-to-cycle, the variance gets added and the accumulated jitter over a period corresponding to an uncoded data bit is the product of the variance of the cycle-to-cycle jitter and the number of pulses per uncoded data bit $(\eta)$, i.e.,

$$
\sigma_{a c c}^{2}=\sigma_{c t c}^{2} \times \eta .
$$

Three different pulse shapes, namely, square, half-sinusoidal and Gaussian are considered in this work. They can be represented mathematically as,

$$
p(t)=\left\{\begin{array}{lll}
\frac{1}{\sqrt{T_{p}}}, & t \in\left[0, T_{p}\right) & \text { (Square) } \\
\beta \sin \left(\pi f_{p} t\right), & \quad t \in\left[0, T_{p}\right) & \text { (Half-sinusoidal) } \\
\gamma \exp \left(-\chi t^{2}\right), & t \in\left[0, T_{p}\right) & \text { (Gaussian) } \\
0 & \text { elsewhere, } &
\end{array}\right.
$$

where $f_{p} \approx 1 / T_{p}, \beta$ and $\gamma$ are normalization constants chosen such that

$$
\int_{0}^{T_{p}}|p(t)|^{2} d t=1 .
$$

The constant $\chi$ is used to control the width of the Gaussian pulse.

Designing a QBEr to track an unknown parameter is a complex process. This optimization problem involves several parameters such as the range and the rms value of the jitter to be 
tracked, the number of states in the QBEr, the sampling rate, etc., some of which are inter-dependent. In order to address this optimization problem in a tangible way, we take the following approach: First, we fix the rms-jitter that can be allowed in the system as well as the complexity of the QBEr that is being used to track this jitter. Given these values, it is straight-forward to see that the sampling window $T_{w}$, hereafter referred to as the search range, and the sampling rate $N / T_{p}$ are inversely related. Under the assumption that known data is present, we use the average signal-to-noise ratio (SNR) at the output of the QBEr as an indicator to asses the trade-off. ${ }^{2}$ The output SNR of the estimator is calculated as follows: The reference signal at the receiver is shifted (in time) according to the estimator output $\breve{\epsilon}_{m}$ at time instant $m$. This signal is then correlated with the received signal corresponding to the $m$-th time instant as shown in Figure 1b. The correlator output is approximated as a Gaussian random variable. The output SNR is calculated as the ratio between the squared value of the mean of this random variable and its variance. After removing the effect of the data, the SNR is averaged over time and over several realizations of the jitter. The QBEr design procedure can be described as follows:

- Choose the cycle-to-cycle jitter $\sigma_{\text {ctc }}$ allowed in the system

- Fix the state complexity of the $Q B E r, Q$, and the pulse shape

$p$

- Given the $\sigma_{c t c}$, choose the resolution $R=T_{p} / N$ with which the jitter is being tracked. This dictates the sampling rate

- For the chosen $R$, find out the search range $W=T_{w}$

- Given $\sigma_{c t c}, N, R$ and $W$, calculate the transition probabilities

- Fix the input $S N R$ (defined in terms of $E_{b} / N_{o}$ ) at a reasonably high value and sample the noisy received signal at $N$ samples per pulse

- Run the estimation algorithm (min*-sum or min-sum), with the sampled data and calculate the average SNR at the output of the estimator

- Repeat for a different $R$

- The value of $W$ (and hence $R$ ) that maximizes the average output SNR is chosen as the winner

Figure 3 shows the result obtained using this algorithm to optimize the QBEr for the following parameters: $0.05 T_{p}$ cycleto-cycle jitter $\left(\sigma_{c t c}=\sigma_{u}\right)$, 7-state estimator and Gaussian pulse shape. The parameter $\chi$ is chosen such that the pulse duration is restricted to $1 \mathrm{~ns}$. It can be seen that restricting the search range to $3 \sigma_{\epsilon}$ which, in turn, corresponds to 8 samples/pulse, maximizes the average output SNR of the estimator. The parameter $\sigma_{\epsilon}$ is related to $\sigma_{c t c}$ or $\sigma_{u}$ by

$$
\sigma_{\epsilon}=\frac{\sigma_{u}}{\sqrt{1-\alpha^{2}}}
$$

Therefore, in the following discussions, unless specified otherwise, the total number of samples per pulse is fixed at 8 and the estimator search range is $3 \sigma_{\epsilon}$.

Figure 4 shows the performance of a low rate coded system concatenated with the QBEr. The low rate code used here is a

\footnotetext{
${ }^{2}$ Note that the mean-squared error can also be used as an indicator
}

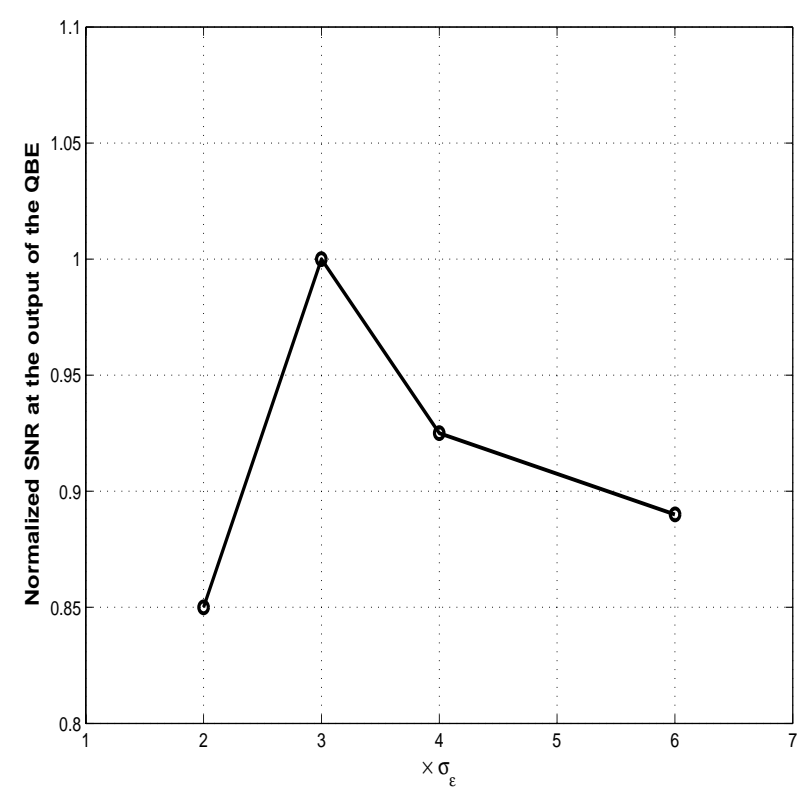

Fig. 3. A QBEr design approach for a fixed cycle-to-cycle jitter, pulse shape, state complexity and high SNR

low rate, parallel concatenated convolutional code, also known as the turbo code. The constituent convolutional codes are 16state, recursive systematic codes. Codes constructed using the methodology described in [7] are used. The code rate is fixed at $1 / 64$. The code rate is related inversely to $\eta$. The estimator trellis has 7 states. Square pulse shape is used. The results reported in the rest of this paper are obtained after performing 10 'over-all' iterations ${ }^{3}$. For comparison, we have plotted the performance of the low rate coded system in the absence of jitter as well. Note that the performance degradation is roughly $2 \mathrm{~dB}$ in $E_{b} / N_{o}$. We have also plotted the performance of the low rate coded system with conventional tracking loop, namely, a delay locked loop (DLL). The bandwidth of the loop filter is a design parameter and, for our study, it is fixed at 10 times smaller than the symbol bandwidth. Note that for the same jitter value, QBEAID has $4 \mathrm{~dB}$ coding gain compared to the performance of the optimized DLL. Finally, we note how the coding gain is traded off with the rms-jitter that is allowed in the system. Typical (maximum) rms-jitter value per symbol duration that is associated with an impulse radio with 'conventional' tracking device, such as the delay-locked loop, is roughly 25ps [8]. The rmsjitter per symbol that is being tracked in our simulation (with code rate $=1 / 64$, cycle-to-cycle jitter $=0.05 T p$ and $\alpha=0.99$ ) is approximately $375 \mathrm{ps}$ which is roughly 15 times more than that which can be tolerated by a 'conventional' system.

In some cases, it might not be necessary to relax the oscillator specification on the phase noise level by a factor of 15 in order to sufficiently drive down the cost. Also in some cases, trading off $2 \mathrm{~dB}$ in coding gain for such relaxation might not be desired. In such a scenario, it is important to understand the trade-off

\footnotetext{
${ }^{3}$ One 'over-all' iteration includes one iteration between constituent codes of the low rate turbo code and one iteration with the estimator.
} 


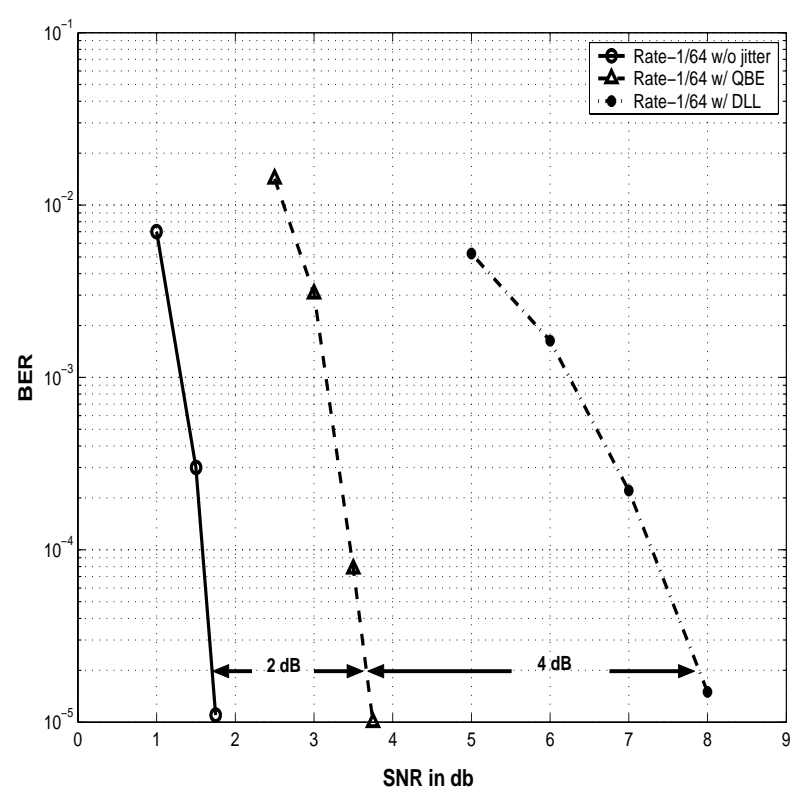

Fig. 4. Simulation results for the QBE-AID scheme (8 samples/symbol, $3 \sigma_{\epsilon}$ search range, 7-state estimator, 375ps rms jitter/symbol, min-star-sum estimation/detection, square pulse)

between the coding gain and the rms-jitter. Figure 5 addresses this issue. Two different algorithms, namely, the min*-sum and the min-sum, are used for the estimation while the $\min ^{*}$-sum algorithm is used for the data detection. The jitter variances that are considered here correspond to $15,7.5$ and 3.75 times more than that which can be handled by a conventional system $[8,9]$, respectively. Square pulse shape is used. Note that as the jitter variance is changed with the sampling rate being fixed, the state complexity of the QBEr decreases from 7 states to 3 . From the simulation results presented in Figure 5, we note that one can track $\sim 4$ times more jitter using the QBE-AID scheme with virtually no additional hardware complexity while losing only a fraction of a $d B$ in $E_{b} / N_{o}$.

The results presented in Figures 4 and 5 which are obtained based on using a square pulse shape may be optimistic. Therefore, the impact of pulse shape on the tracking performance was investigated and the results are shown in Table 1. Three different pulse shapes are considered. The rms-jitter is fixed at $375 \mathrm{ps}$ and 7-state estimator is used to track the jitter. The required BER at the receiver is fixed at $10^{-5}$. Results are shown both for the min*-sum and min-sum estimation algorithms. Note that a performance loss of at least $1 \mathrm{~dB}$ in $E_{b} / N_{o}$ is incurred as the pulse shape is changed from square to Gaussian, irrespective of the estimation algorithm.

In the results discussed thus far, it was assumed that the jitter model (pole location of the filter) was known at the receiver. However, in practice, the pole location needs to be estimated before being used in calculating the transition probabilities for the QBEr. Therefore, it is important to know how sensitive the tracking system is to this estimation error. It is desirable that the tracking algorithm is insensitive for a wide range of mismatch in $\alpha$. From the simulation results we found that even for a mis-

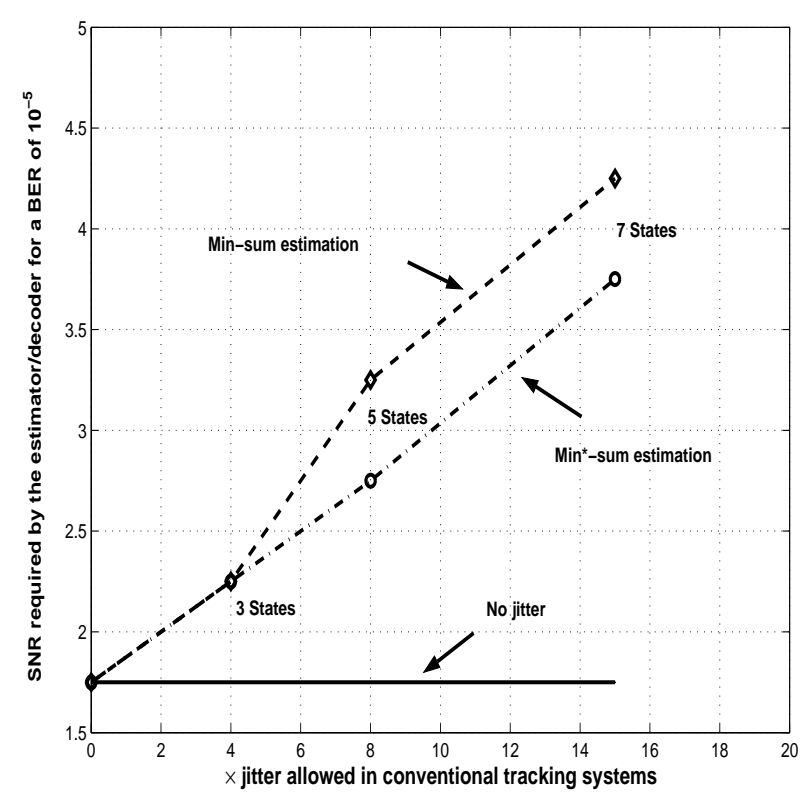

Fig. 5. Simulation results for the QBE-AID scheme for various cycle-to-cycle jitter values ( 8 samples/pulse, $3 \sigma_{\epsilon}$ search range, 7 -state estimator: $375 \mathrm{ps}$ rms jitter/symbol, 5-state estimator: $187.5 \mathrm{ps} \mathrm{rms}$ jitter/symbol, 3-state estimator: 93.75ps rms jitter/symbol, min-star-sum decoding, square pulse, rate-1/64 turbo code).

\begin{tabular}{|c|c|c|}
\hline \hline Pulse Shape & Min*-sum Estimation & Min-sum Estimation \\
\hline Square & $3.75 \mathrm{~dB}$ & $4.25 \mathrm{~dB}$ \\
Half-sine & $4.00 \mathrm{~dB}$ & $4.75 \mathrm{~dB}$ \\
Gauss & $4.75 \mathrm{~dB}$ & $5.50 \mathrm{~dB}$ \\
\hline \hline
\end{tabular}

TABLE I

PERFORMANCE OF THE QBE-AID AS A FUNCTION OF PULSE SHAPE ( 8 SAMPLES/PULSE, $3 \sigma_{\epsilon}$ SEARCH RANGE, 7-STATE ESTIMATOR, 375PS RMS JITTER/SYMBOL, MIN-STAR-SUM DECODING, RATE-1/64 TURBO CODE). THE TARGET BER IS $10^{-5}$.

match of $0.2(|\alpha-\hat{\alpha}|=0.2)$ the loss in $E_{b} / N_{o}$ to maintain a BER of $10^{-5}$ is less than $0.5 \mathrm{~dB}$. This indicates that the $\mathrm{QBEr}$ is very robust to the mismatch in the jitter model.

\section{CONCLUSIONS AND FUTURE WORK}

We have proposed a novel scheme for timing estimation that can be used to relax the constraint on the clocks used in coded UWB systems. The proposed estimator exchanges soft information with the decoder iteratively in order to refine the estimated values. This scheme can be used to trade the complexity of the digital backend of the UWB receiver with the specification on the oscillator. In this work, only the estimation and compensation of the short term instability of the clock are studied. Estimation of the long term instability of the oscillators, namely the drift, is an interesting and important problem that we are currently studying. 


\section{APPENDIX}

A connection is established between the maximum $a$ posteriori probability based expectation-maximization (MAPEM) and the min-sum estimation algorithm for the QBEr. Typically, the maximum likelihood EM (ML-EM) algorithm is used in estimation of unknown parameters in the absence of complete data. In tracking, the received signal can be treated as incomplete data while the received signal along with the coded symbols being the complete data. Then, a MAP based EM can be derived whose outputs will be the soft information on the jitter. For simplicity, we choose to use natural logarithm in this derivation.

Let the received signal be re-written as

$$
\underline{r}=\underline{b s}(\underline{\epsilon})+\underline{n} .
$$

Under the assumption that the model for the unknown jitter is discretized, which is the the key for the development of the QBE, the MAP-EM can be written as,

$$
\begin{aligned}
& \text { E-step: Compute } Q\left(\underline{\hat{\epsilon}}, \underline{\hat{\epsilon}}^{k}\right)=\mathrm{E}_{\underline{b}}\left\{[\log \operatorname{Pr}(\underline{r} \mid \underline{\hat{\epsilon}}, \underline{b})] \mid \underline{r}, \underline{\hat{\epsilon}}^{k}\right\} \\
& \text { M-step: } \quad \text { Solve } \underline{\breve{\epsilon}}^{(k+1)}=\arg \max _{\hat{\underline{\epsilon}}}\left[Q\left(\underline{\hat{\epsilon}}, \underline{\hat{\epsilon}}^{k}\right)+\log \operatorname{Pr}(\underline{\hat{\epsilon}})\right] .
\end{aligned}
$$

The only difference between the ML-EM and the MAP-EM is the presence of $a$-priori probabilities $\operatorname{Pr}(\underline{\hat{\epsilon}})$ in the M-step. This is an iterative procedure where the E-step and the M-step are iterated till the estimated values $\underline{\hat{\epsilon}}$ converge. The iteration between the E-step and the M-step is referred to as self iteration and is indexed by $k$. Note that MAP-EM is a sequence detection/estimation algorithm.

The MAP based estimation rule for estimating $\hat{\epsilon}_{m}$ implied by the MAP estimation of $\underline{\hat{\epsilon}}$ is given by

$$
\begin{aligned}
& \breve{\epsilon}_{m}^{(k+1)}=\arg \max _{\hat{\epsilon}_{m}} \max _{\hat{\hat{\epsilon}}: \hat{\epsilon}_{m}} \operatorname{Pr}\{\underline{\hat{\epsilon}} \mid \underline{r}\}, \\
& \equiv \arg \max _{\hat{\epsilon}_{m}} \max _{\hat{\hat{\epsilon}}: \hat{\epsilon}_{m}} \log \operatorname{Pr}\{\underline{\hat{\epsilon}} \mid \underline{r}\}, \\
& =\arg \max _{\hat{\epsilon}} \max _{\hat{\hat{\epsilon}}: \hat{\epsilon}} \log \operatorname{Pr}\{\underline{r} \mid \hat{\hat{\epsilon}}\} \operatorname{Pr}\{\underline{\hat{\epsilon}}\}, \\
& =\arg \max _{\hat{\epsilon}_{m}} \max _{\hat{\hat{\epsilon}}: \hat{\epsilon}_{m}}\left(\mathrm{E}_{\underline{b}}[\log \operatorname{Pr}\{\underline{r} \mid \underline{b}, \underline{\hat{\epsilon}}\}]\right. \\
& +\log \operatorname{Pr}\{\underline{\hat{\epsilon}}\}), \\
& \approx \arg \max _{\hat{\epsilon}_{m}} \max _{\hat{\epsilon}: \hat{\epsilon}_{m}}\left(\left[\sum_{\underline{b}}\left\{\sum_{i} \log \operatorname{Pr}\left\{\underline{r}_{i} \mid b_{i}, \hat{\epsilon}_{i}\right\}\right\}\right.\right. \\
& \left.\left.\operatorname{Pr}\left\{\underline{b} \mid \underline{r}, \underline{\hat{\epsilon}}^{k}\right\}\right]+\sum_{i} \log \operatorname{Pr}\left\{\hat{\epsilon}_{i} \mid \hat{\epsilon}_{i-1}\right\}\right), \\
& =\arg \max _{\hat{\epsilon}_{m}} \max _{\hat{\hat{\epsilon}}: \hat{\epsilon}_{m}}\left(\left[\sum _ { i } \left\{\sum_{b_{i}} \log \operatorname{Pr}\left\{\underline{r}_{i} \mid b_{i}, \hat{\epsilon}_{i}\right\}\right.\right.\right. \\
& \left.\left.\left.\operatorname{Pr}\left\{b_{i} \mid \underline{r}_{i}, \hat{\epsilon}_{i}^{k}\right\}\right\}+\log \operatorname{Pr}\left\{\hat{\epsilon}_{i} \mid \hat{\epsilon}_{i-1}\right\}\right]\right) .
\end{aligned}
$$

The first line of (16) is one way of solving (6). Similarly, (16.4) is same as (15). Therefore, the MAP-EM can be performed on the trellis shown in Figure 2b. Since the trellis is used for combining and marginalization, the need for self iteration is also eliminated. Therefore, the min-sum estimation algorithm for the QBEr can be viewed as the MAP-EM.

\section{REFERENCES}

[1] C. Berrou , A. Glavieux, and P. Thitimajshima, "Near Shannon limit errorcorrecting coding and decoding: turbo-codes," in Proc. ICC'93, Geneva, Switzerland, May 1993, pp. 1064-1070.

[2] K. M. Chugg et. al, Iterative Detection: Adaptivity, Complexity Reduction, and Applications, Kluwer Academic Publishers, MA, 2001.

[3] A. Anastasopoulos, "Adaptive Soft-In Soft-Out Algorithms and Iterative Detection,” Ph.D. Thesis, University of Southern California, 1999.

[4] A, Demir, A. Mehrotra and J. Roychowdhury, "Phase noise in oscillators: a unifying theory and numerical methods for characterization," IEEE Trans. on circuits and systems-I: Fundamental Theory and Applications, vol. 47, No. 5, pp. 655-674, May 2000.

[5] J. G. Proakis, Digital Communications, McGraw-Hill , Inc., NY 1995.

[6] K. Lerdsuwanakij, "On Receivers for Multipath Fading and Random Phase Channels," Ph.D. Thesis, University of Southern California, 2000.

[7] D. Thirupathi and K. M. Chugg, "A simple construction of low rate convolutional codes with application to low rate turbo-like code design," in Proc. Globecom 2002.

[8] W. M. Lovelace and J. K. Townsend, "The effects of timing jitter and tracking on the performance of impulse radio," IEEE Journ. on Select. Areas. Commun., vol. 20, no. 9. pp.1646-1651, Dec. 2002.

[9] I. O’Donnell, M. Chen, S. Wang and R. W. Broderson, "An integrated, low power, ultra-wide band transceiver architecture for low rate, indoor wireless systems," in Proc. IEEE CAS workshop on Communications and Networking 2002 